\title{
Ventral T-1 Neurinoma Removed Via Hemilaminectomy Without Costotransversectomy
}

\author{
-Case Report- \\ Yukihide Kanemoto, Hideyuki OHNISHI, Naoki Koshimae, \\ Tomonori YAMADA, Yeong-Jin KIM, Kunihiko KoBITSU, \\ and Yasushi MOTOYAMA \\ Department of Neurosurgery, Osaka Police Hospital, Osaka
}

\begin{abstract}
A 39-year-old male presented with a spinal neurinoma originating from the T-1 anterior root and located ventral to the spinal cord. The tumor was removed by hemilaminectomy with only partial facetectomy without costotransversectomy. No stabilization was necessary, and no complications secondary to surgery occurred. Costotransversectomy is not necessary for neurinoma ventral to the spinal cord within the spinal canal at $T-1$ level because the transverse process protrudes more laterally and the spinal canal of the $T-1$ vertebra is wider than at other thoracic levels.
\end{abstract}

Key words: posterolateral approach, spinal neurinoma, anterior root, surgery

\section{Introduction}

Spinal neurinomas originating from the anterior roots account for less than $5 \%$ of all spinal neurinomas. ${ }^{10,11)}$ As the origins of these neurinomas are located on the ventral spinal cord and within the spinal canal without enlargement of the neuroforamen, it is difficult to decide on a surgical approach. Various approaches to lesions at the thoracic levels include the anterior thoracic, ${ }^{1,4,5,7-9,15,16,18)}$ posterior, posterolateral, ${ }^{13,14,17)}$ and lateral extrapleural approaches. ${ }^{3,7,12)}$ The anterior approach provides direct access to the ventral surface of the spinal cord. However, the operative procedure is complex and difficult because of the deep, narrow corridor and necessity for vertebrectomy. Moreover, there are potentially formidable sequelae, ${ }^{2,6)}$ such as cerebrospinal fluid (CSF) leakage and respiratory dysfunction. Recently, posterolateral approaches with costotransversectomy have frequently been used for lesions ventral to the spinal cord. ${ }^{13,14,17)}$ The posterolateral approach with dentate ligament section and spinal cord rotation provides excellent ex- posure to the lateral surface of the spinal cord, ${ }^{13)}$ but most posterolateral approaches at the thoracic levels require transverse incision of the paraspinous muscles and costotransversectomy.

We present a case of a spinal neurinoma originating from the T-1 anterior root and located ventral to the spinal cord which was treated by hemilaminectomy with only partial facetectomy, and discuss whether costotransversectomy is indispensable at the T-1 level using the posterolateral approach to lesions ventral to the spinal cord.

\section{Case Report}

A 39-year-old male had suffered from chest oppression, and numbness and motor weakness of the lower extremities on both sides for about 2 years before admission. His condition had gradually deteriorated. He had a history of drug abuse since the age of 18 years. The patient was transferred to a local hospital when he was unable to walk alone and suffered from confusion due to withdrawal symptoms. The diagnosis was a spinal tumor at the T-1 


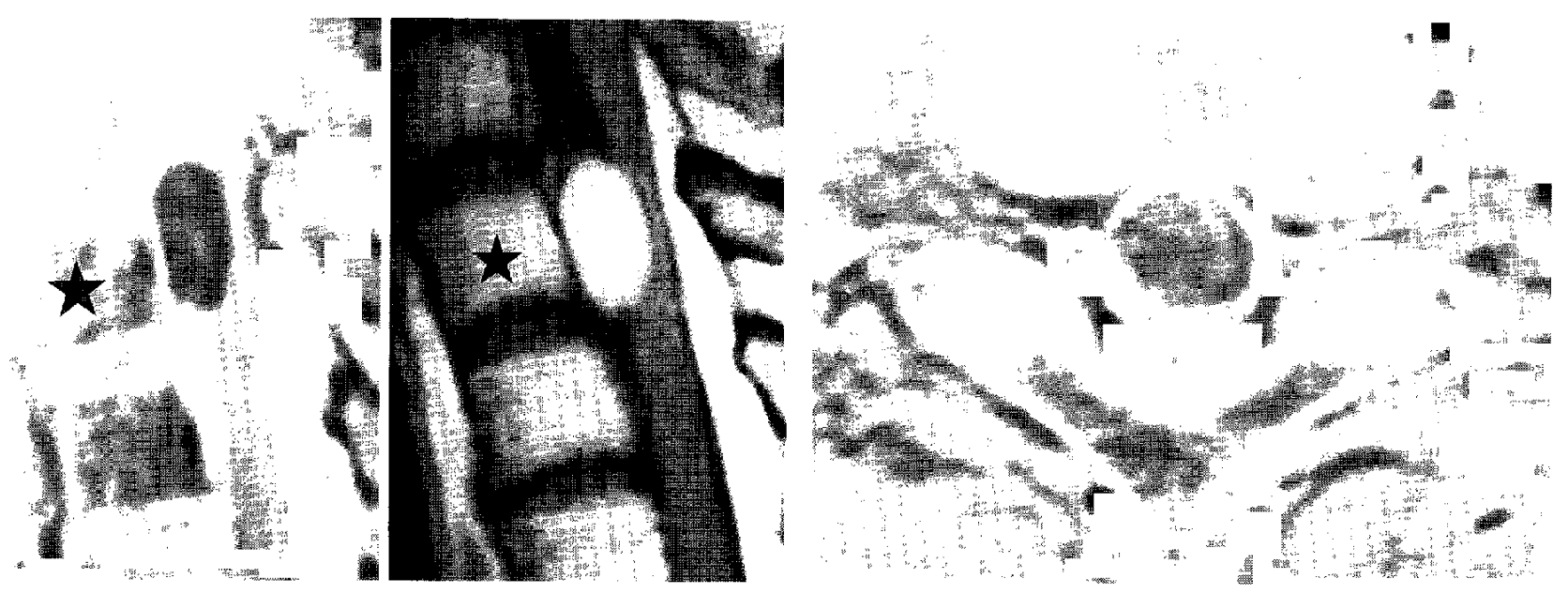

Fig. 1 left: $T_{1}$-weighted magnetic resonance (MR) image with administration of gadoliniumdiethylenetriaminepenta-acetic acid demonstrating a homogeneously enhanced $2 \mathrm{~cm}$ intradural extramedullary mass severely compressing the left of the spinal cord dorsally at the T-1 level (star). center: Repeat MR image revealing a slight change in the tumor location, compared with Fig. 1 left. Star indicates T-1 thoracic vertebral body. right: Axial MR image showing the severely deformed left of the spinal cord compressed dorsally by the round tumor at the T-1 level.

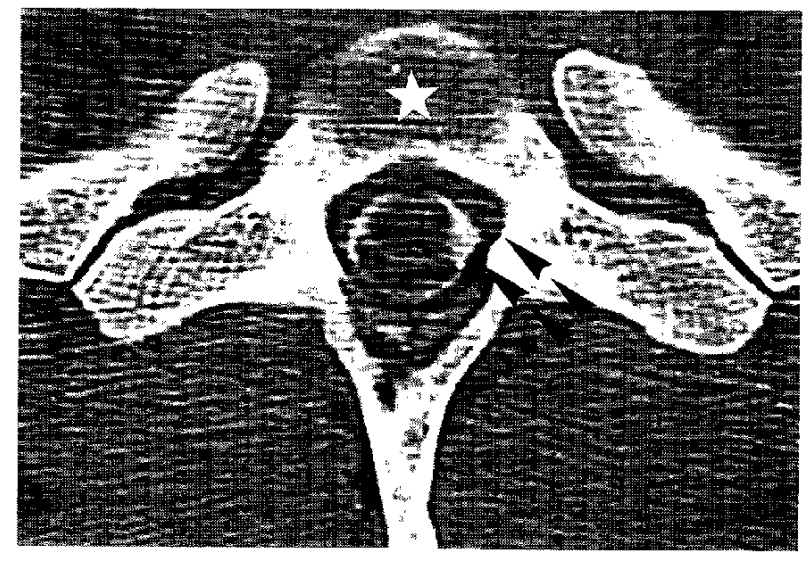

Fig. 2 Computed tomography scan after myelography revealing a thin rim of contrast medium surrounding the margin of the tumor. Costotransversectomy at the T-1 level is not necessary to obtain direct access to the ventrolateral surface of the spinal cord (arrowheads). Star indicates T-1 thoracic vertebral body.

level. The patient was referred to our hospital for management of the lesion.

Neurological examination demonstrated grossly complete anesthesia of lower than T-3 dermatome, severe disturbance of deep sensation of the lower extremities, and moderate paraparesis. Bladderbowel functions were grossly normal.
Radiography showed no abnormalities. Magnetic resonance (MR) imaging demonstrated a $2 \mathrm{~cm}$ intradural extramedullary mass severely compressing the left spinal cord dorsally at the T-1 level, which appeared as isointense on $\mathrm{T}_{1}$-weighted images with homogeneous enhancement after administration of gadolinium-diethylenetriaminepenta-acetic acid. Repeat MR imaging revealed a slight change in tumor location, which indicated mobilization of the tumor in the spinal canal (Fig. 1). Computed tomography (CT) after myelography revealed a thin rim of contrast medium surrounding the margin of the tumor located ventral and right of the spinal cord, which suggested neurinoma rather than meningioma (Fig. 2).

The patient underwent total removal of the tumor in the right-side up, three-quarter position under general anesthesia. Following the longitudinal midline skin incision, the paraspinous muscles on the right were dissected subperiosteally from the spinous process to the T-1 transverse process. T-1 hemilaminectomy with skeletonization of the partial C-7 and T-2 hemilaminae was performed. The base of the spinous process was skeletonized obliquely to obtain space for dorsal rotation of the spinal cord. The T-1 pedicle and parts of the C7-T1 and T1-2 facets were skeletonized to provide direct access to the lateral surface of the spinal cord and to allow T-1 root mobilization (Fig. 3). The tumor originated from the T-1 anterior root, which was located in the epiarachnoid space, and was completely removed 


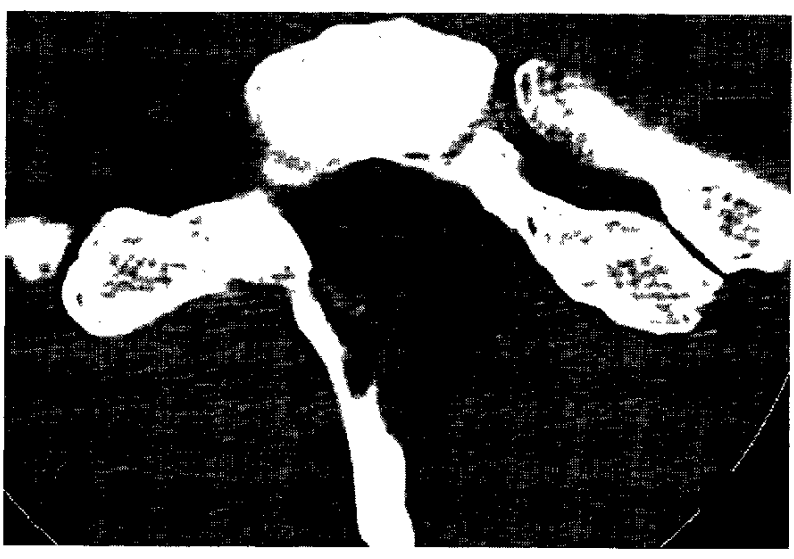

Fig. 3 Postoperative computed tomography scan demonstrating the bone removal area sparing the transverse process at the T-1 level.

with no forced retraction of the spinal cord. Neither internal nor external stabilization was used. The histological diagnosis was neurinoma.

The preoperative symptoms and signs subsided completely, except for very slight motor weakness of the left lower extremity and chest oppression one month after surgery. No complications secondary to surgery, such as root signs of T-1, instability, or CSF leakage, occurred.

\section{Discussion}

The present case of ventrally located spinal neurinoma at the T-1 level could be safely removed by the posterolateral approach without requiring potentially risky procedures to provide direct access to the ventrolateral surface of the spinal cord.

We would like to emphasize two points that made costotransversectomy unnecessary. Firstly, the tumor was identified as a neurinoma preoperatively by CT myelography and serial MR imaging. Therefore, it was unnecessary to treat the ventral site dura mater. Secondly, the T-1 vertebra has morphological characteristics that are different from those of other thoracic vertebrae. The transverse process protrudes more laterally and the spinal canal is wider at the T-1 level than those of other thoracic vertebrae. Therefore, costotransversectomy at the T-1 level is not necessary to obtain direct access to the ventrolateral surface of the spinal cord, although indispensable at the levels of other thoracic vertebrae, as seen by comparing Figs. 2 and 4 .

In our case, skeletonization of a limited bone area, including the hemilamina, and the medial half of the facets and the pedicle, allowed considerable mobilization of the affected root, and sectioning of the

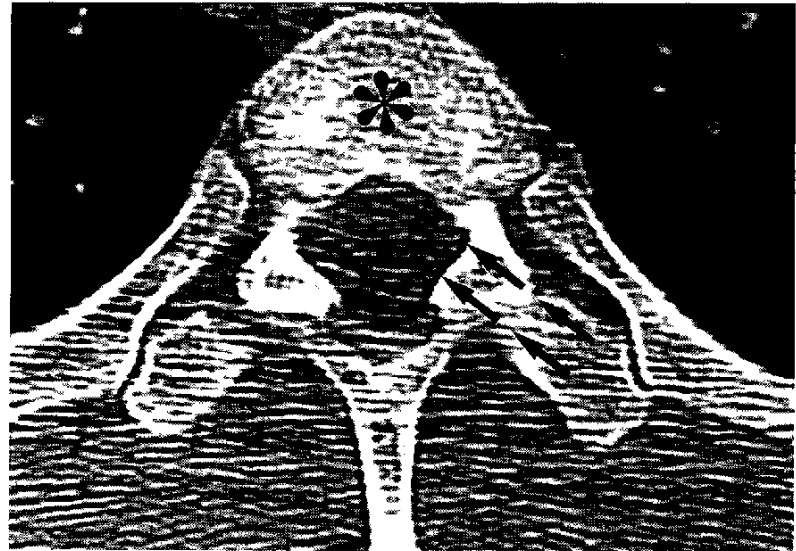

Fig. 4 Computed tomography scan after myelography at the $T-3$ level showing the transverse process protrudes more dorsally and the spinal canal is narrower at the $T-3$ vertebra (asterisk) compared to those at the T-1 level (Fig. 2). Transversectomy is indispensable at the $T-3$ vertebra to obtain direct access to the ventrolateral surface of the spinal cord (arrows).

dentate ligament enabled safe dorsal rotation of the spinal cord. Thereafter, direct access to the ventrolateral surface of the spinal cord was obtained using limited procedures without costotransversectomy. Internal and external stabilization was unnecessary because of the limited area of bone removal. Moreover, the omission of costotransversectomy reduces the degree of paraspinous muscle transection necessary. In our case, paraspinous muscle transection was not required because the muscle mass did not interfere with access to the lateral surface of the spinal cord. Therefore, potential complications caused by paraspinous muscle transection were avoided.

The posterolateral approach without costotransversectomy can be used for neurinoma ventral to the spinal cord within the spinal canal at the T-1 level because of the morphological characteristics of the T-1 vertebra. Consequent limitation of bone removal prevents various complications.

\section{References}

1) Anderson TM, Mansour KA, Miller JI Jr: Thoracic approaches to anterior spinal operations: anterior thoracic approaches. Ann Thorac Surg 55: 14471452, 1993

2) Assietti R, Kibble MB, Bakay RAE: Cerebrospinal fluid fistula to the pleural cavity: Case report and literature review. Neurosurgery 33: 1104-1108, 1993 
3) Benzel EC: The lateral extracavitary approach to the spine using the three-quarter prone position. $J$ Neurosurg 71: 837-841, 1989

4) Charles R, Govender S: Anterior approach to the upper thoracic vertebrae. J Bone Joint Surg [Br] 71: 8184, 1989

5) Darling GE, McBroom R, Perrin R: Modified anterior approach to the cervicothoracic junction. Spine 13: 1519-1521, 1995

6) Diaz P, Maillo A, Hernandez J, Morales F, Jimenez MF, Moreta JA, Martin JA: Subarachnoid-pleural fistula as a complication of the lateral-extracavitary approach to thoracic intraspinal neurinoma. Spine 13: 1515-1518, 1995

7) Fessler RG, Dietze DD Jr, Mac Millan M, Peace D: Lateral parascapular extrapleural approach to the upper thoracic spine. J Neurosurg 75: 349-355, 1991

8) Fielding JW, Stillwell WT: Anterior cervical approach to the upper thoracic spine. A case report. Spine 1: 158-161, 1976

9) Frantz PT, Battagliani JW: Subarachnoid-pleural fistula. Unusual complication of thoracotomy. J Thorac Cardiovasc Surg 79: 873-875, 1980

10) Gautier-Smith PC: Clinical aspects of spinal neurofibromas. Brain 90: 359-394, 1967

11] Hori T, Takakura $K$, Sano K: Spinal neurinomas: Clinical analysis of 45 cases. Neurol Med Chir (Tokyo) 24: 471-477, 1984

12) Maiman DJ, Larson SJ, Luck E, El-Ghatit A: Lateral extracavitary approach to the spine for thoracic disc herniation: Report of 23 cases. Neurosurgery 14: $178-182,1984$

13) Martin NA, Khanna RK, Batzdorf U: Posterolateral cervical or thoracic approach with spinal cord rotation for vascular malformations or tumors of the ventrolateral spinal cord. I Neurosurg 83: 254-261, 1995

14) McCormick PC: Surgical management of dumbbell and paraspinal tumars of the thoracic and lumbar spine. Neurosurgery 38: 67-75, 1996

15) Micheli LJ, Hood RW: Anterior exposure of the cervicothoracic spine using a combined cervical and thoracic approach. J Bone Joint Surg [Am] 65: 992997, 1983

16) Raynor RB, Weiner R: Transthoracic approach to an intramedullary vascular malformation of the thoracic spinal cord. Neurosurgery 10: 631-634, 1982

17) Steck JC, Dietze DD, Fessler RG: Posterolateral approach to intradural extramedullary thoracic tumors. J Neurosurg 81: 202-205, 1994

18) Sundaresan N, Shah J, Foley KM, Rosen G: An anterior surgical approach to the upper thoracic vertebrae. J Neurosurg 61: 686-690, 1984

Address reprint requests to: Y. Kanemoto, M.D., Department of Neurosurgery, Nara Prefectural Gojo Hospital, 197 Nohara-cho, Gojo, Nara 637-0034, Japan. 